Ann. Zootech., I971, 20 (3), 275-290.

\title{
CONSERVATION PAR ENSILAGE OU DÉSHYDRATATION D'UNE ORGE ET D'UN MAÏS IMMATURES
}

\author{
Ph. GOUET ( ${ }^{1}$ ), Y. RIOU (1) et Nathalie BOUSSET-FATIANOFF \\ avec la collaboration technique de Nadine Rıou-Mounous, \\ Christiane Bìs et Marie-France DORBE \\ Laboratoire de Recherches sur la Conservation et l'Efficacité des Aliments, \\ Centre national de Recherches zootechniques, I.N.R.A., \\ 78 - Jouy-en-Josas
}

\section{RÉSUMÉ}

On a comparé durant deux années consécutives les rendements d'une orge (Rika) et d'un maìs $(I . N . R . A .260)$ récoltés au stade vitreux une à quatre semaines avant maturité, à ceux obtenus au stade habituel de récolte; l'orge est récoltée avec 20 à $30 \mathrm{~cm}$ de paille et l'épi de maïs avec ses spathes. Ces céréales sont conservées, soit par ensilage avec $\left(U^{+}\right)$ou sans urée (U $\left.{ }^{-}\right)$ dans des silos étanches en butyl, soit par déshydratation à basse température ( $130^{\circ} \mathrm{C}$ ) ; on analyse dans les premières les produits de la fermentation bactérienne et on établit pour toutes les bilans de la conservation.

Pour les deux céréales, les rendements en matière sèche (MS) de grain obtenus à ce stade vitreux, sont inférieurs, selon les essais de 8,8 p. Ioo à 30 p. I oo à ceux obtenus au stade mature. La matière sèche du grain immature représente de $72, \mathrm{I}$ p. Ioo à $79 \mathrm{p}$. Ioo de la matière sèche totale récoltée. Le rendement $\mathrm{MS}$ totale récoltée varie de $-8 \mathrm{p}$. 100 à $+24,8 \mathrm{p}$. roo. On conclut que la verse, fréquente chez l'orge, et l'absence de machines adaptées rendent difficile une coupe haute régulière et provoquent des pertes importantes.

Les proportions des différents composants morphologiques (paille, grain, barbillons, rachis, rafle, pédoncule) sont présentées ainsi que leur composition chimique (matière organique, cellulose Weende, azote).

Dans les ensilages, les concentrations en métabolites fermentaires (acide lactique, acides gras volatils, alcool, $\mathrm{N}_{-} \mathrm{NH}_{3}$ ) sont faibles et caractéristiques des ensilages à teneur élevée en matière sèche.

L'ensilage d'orge est toutefois plus sensible à la fermentation butyrique que le maîs et les nombres de spores de Clostridium fermentant le lactate ou protéolytiques y atteignent souvent $\mathbf{1}^{\circ}$ à 5. $10^{7}$ par gramme. L'addition d'urée se traduit par une augmentation du taux d'ammoniaque et un accroissement significatif de l'intensité fermentaire. Ceci a pour conséquence de doubler les pertes de matière organique dans les ensilages d'orge (Io p. Ioo).

Dans l'ensilage d'épis de maïs, ces pertes restent les mêmes dans $U$ - et $U+(2 p$. Ioo à 3 P. IOO).

La déshydratation à basse température, aisée pour l'orge, l'est moins pour le maïs en raison des différences importantes dans les taux de matière sèche et les vitesses de dessication de ses constituants morphologiques. Cette technique permet d'obtenir un produit stable avec des pertes minimes, mais son coût est élevé.

(1) Adresse actuelle : Laboratoire de Microbiologie, Centre de Recherches de Clermont-Ferrand, 63 - Saint-Genes-Champanelle. 


\section{INTRODUCTION}

L'utilisation zootechnique des céréales récoltées avant maturité complète du grain et avec une partie de la plante (paille, spathes, rafle) puis conservées par ensilage ou déshydratation peut être appelée à se développer si les problèmes techniques et économiques posés par ce nouveau procédé sont résolus. C'est ainsi que la faible teneur en matières azotées de ces plantes implique une complémentation qui peut être faite avec de l'urée, mais l'addition de celle-ci risque de modifier l'intensité (OWENS, MEISKE, GOODRICH, I969) et l'orientation des fermentations ainsi que les pertes qui leur sont liées. D'autre part, le comportement de l'orge ensilée avec sa tige est pour le moment assez peu connu (EDwards, Donaldson, Mc Gregor, rg68) surtout quand le taux de matière sèche atteint 50 p. Ioo. La déshydratation et l'ensilage d'épis de maïs n'ont pas fait non plus l'objet de recherches technologiques détaillées. Cette étude se propose donc principalement de déterminer les aptitudes à la déshydratation et à l'ensilage (avec ou sans urée) d'une orge récoltée en coupe hatte et d'épis de maïs broyés avec leurs spathes puis accessoirement de mesurer les rendements agronomiques de ces céréales. L'étude de l'utilisation zootechnique des aliments ainsi conservés est rapportée dans un autre mémoire (ZELTER, CHARLET-LÉRY TISSERAND, I97I).

\section{MATÉRIEL, E'T MÉTHODE}

$$
\text { 1. - Récolte }
$$

Au cours de deux années consécutives (1967-1968) une orge (variété Rika) et un maïs $(I . N . R . A .260)$ sont récoltés une à quatre semaines avant maturité. A chaque récolte, on mesure les rendements aux stades immature et mature ainsi que les proportions des différents composants morphologiques de ces plantes (grain, paille, rachis, spathes, rafles) et leur composition chimique (matières minérales, organique, cellulose Weende, azote total).

La fumure, les doses et dates de semis sont présentées dans le tableau $\mathbf{I}$.

Orge.

En $1967,3,89$ ha d'orge sont coupés les I I ct I 2 juillet dix jours avant la récolte à la moissonneuse-batteuse. On utilise une récolteuse à fourrage dont la barre est rehaussée de façon à couper à $70-75 \mathrm{~cm}$ du sol. Les épis et la paille sont ensuite hachés à poste fixe avec une ensileuse munie de 6 couteaux.

En $1968,3,86$ ha de la même variété sont récoltés les 23 et 24 juillet avec une récolteusehacheuse chargeuse hachant très finement $(\mathrm{t}-2 \mathrm{~cm})$. La barre de coupe qui a été rehaussée pour couper au maximum à $65 \mathrm{~cm}$ ne peut plus, dans ces conditions, couper au-dessous de $30 \mathrm{~cm}$. L'orge restante $(5,53 \mathrm{ha})$ est moissonnée huit jours après.

Mais.

Du 25 au 28 septembre 1967 , douze jours avant maturité, le maïs $(2,39$ ha) est récolté pour moitié à la main, ce qui permet de conserver tous les spathes et pédoncules, et pour le reste avec un corn-picker dont les rouleaux sont desserrés au maximum; la plupart des spathes sont néanmoins éliminées. En $\mathrm{I} 968$, cette céréale est récoltée ( $\mathrm{I}, 76 \mathrm{ha}$ ) entièrement à la main du 2 au 5 octobre. Le maïs restant $(0,24$ ha en 1967 et 0,84 ha en 1968$)$ est récolté le 9 novembre avec un corn-picker. 
TABIEAU I

Fumure, dose et date de semis

\begin{tabular}{|c|c|c|c|c|c|}
\hline & \multirow{2}{*}{$\begin{array}{c}\text { Date et dose } \\
\text { de semis }\end{array}$} & \multicolumn{4}{|c|}{ Fumure } \\
\hline & & Fumier & $\begin{array}{c}\mathrm{N} \\
\text { (unités) }\end{array}$ & $\begin{array}{c}\mathrm{P}_{2} \mathrm{O}_{5} \\
\text { (unités) }\end{array}$ & $\begin{array}{c}\mathrm{K}_{2} \mathrm{O} \\
\text { (unités) }\end{array}$ \\
\hline \multirow{2}{*}{ Orge Rika } & $\begin{array}{l}6-7 / 3 / 1967 \\
115 \mathrm{~kg} / \mathrm{ha}\end{array}$ & & $\begin{array}{r}50 \\
18 / 3\end{array}$ & $\begin{array}{c}65 \\
\text { oct. } 66\end{array}$ & $\begin{array}{c}65 \\
\text { oct. } 66\end{array}$ \\
\hline & $\begin{array}{l}6-8 / 3 / 1968 \\
110 \mathrm{~kg} / \mathrm{ha}\end{array}$ & & $\begin{array}{r}40 \\
27 / 4\end{array}$ & $\begin{array}{c}65 \\
\text { oct. } 67\end{array}$ & $\begin{array}{c}65 \\
\text { oct. } 67\end{array}$ \\
\hline \multirow{2}{*}{ 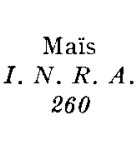 } & $\begin{array}{r}27 / 4 / 1967 \\
27 \mathrm{~kg} / \mathrm{ha}\end{array}$ & $\begin{array}{l}40 \mathrm{t} / \mathrm{ha} \\
\text { mars } 67\end{array}$ & $\begin{array}{l}120 \\
22 / 4\end{array}$ & $\begin{array}{c}65 \\
\text { oct. } 66\end{array}$ & $\begin{array}{c}65 \\
\text { oct. } 66\end{array}$ \\
\hline & $\begin{array}{r}23 / 4 / 1968 \\
27 \mathrm{~kg} / \mathrm{ha}\end{array}$ & $\begin{array}{l}10 \mathrm{t} / \mathrm{ha} \\
\text { sept. } 67\end{array}$ & $\begin{array}{l}140 \\
18 / 4\end{array}$ & $\begin{array}{c}65 \\
\text { août } 67\end{array}$ & $\begin{array}{c}65 \\
\text { août } 67\end{array}$ \\
\hline
\end{tabular}

\section{2. - Ensilage}

Les traitements expérimentés pour les deux céréales, le nombre de répétitions, les quantités ensilées, les taux de matière sèche, les proportions d'urée employées sont rapportées dans le tableau 2.

TABLEAU 2

Traitements expérimentés

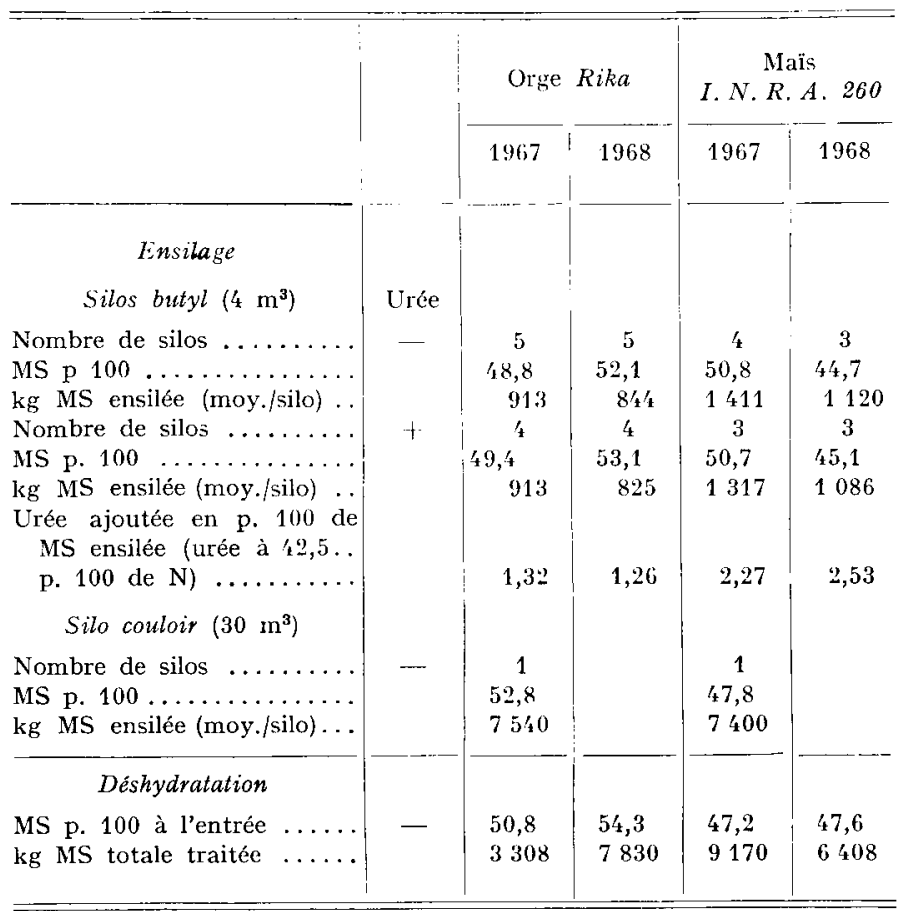


Orge.

L'orge est ensilée comparativement en silo couloir et butyl en I967 et en silo butyl uniquement en 1968. Les silos en butyl sont des cylindres de $4 \mathrm{~m}^{3}$ ( $\mathrm{r}, 55 \mathrm{~m}$ de diamètre $\times \mathrm{r}, 80 \mathrm{~m}$ de hauteur).

Le tassement $y$ est effectué par piétinement et, après fermeture, on pratique un vide de $35 \mathrm{~cm}$ de $\mathrm{Hg}$ qui réduit le volume des silos d'environ io p. Ioo.

En 1967, les silos sont remplis par couple, sans urée (U -) avec urée (U +). En Ig68 aucun appariement n'est effectué et tous les silos correspondant à un même traitement sont remplis en même temps. Pour assurer une répartition homogène et proportionnelle de l'urée les silos sont remplis à l'aide de bacs de $50 \mathrm{~kg}$ auxquels on ajoute une dose appropriée (I,29 p. Ioo MS).

En 1967, quinze tonnes de produit frais sont ensilés dans un silo couloir dont les murs sont faits de plaques de béton juxtaposées verticalement et doublées intérieurement par un film de polyéthylène. Après tassement on charge l'ensilage avec une poche en butyl contenant 40 à $60 \mathrm{~cm}$ d'eau.

\section{Mais.}

En 1967 les panouilles sont broyées deux fois dans un broyeur à marteaux de façon à ce que tous les grains soient écrasés.

En I968, les panouilles sont hachées à poste fixe avec l'ensileuse à couteaux qui a servi pour l'orge et passent une seule fois dans un broyeur à marteaux. Lc maïs est ensilé dans les deux types de silos précédemment décrits, mais le vide n'y est pas effectué. L'urée est ajoutée de la même façon que pour l'orge, mais à la dose de 2,4 p. Ioo du produit sec.

Pour chaque céréale et chaque traitement l'évolution des températures est contrôlée dans un ou deux silos au moyen de deux thermistances disposées aux tiers supérieur et inférieur.

\section{Durée de conservation.}

Elles s'échelonnent de 76 à 346 jours selon la céréale et l'année (fig. 5). En I967, les échantillons sont prélevés à la sonde sur toute l'épaisseur de l'ensilage, et les silos sont sondés par couple $\left(U^{-}, U^{+}\right)$, quelques jours avant la période d'utilisation. Par contre, en I968, cette période s'étendant pour l'ensilage de maïs sur plusieurs mois, les silos sont sondés à la même date (346 jour) pour ne pas introduire de durées inégales de conservation comme facteur de variation supplémentaire.

$$
\text { 3. - Déshydratation }
$$

Orge.

Après avoir subi le même conditionnement que la partie destinée à être ensilée, cette céréale est déshydratée dans un appareil Scolari. La température de contact est de $130^{\circ} \mathrm{C}$ et la durée de séchage varie de 30 à 40 minutes (tabl. 2).

Mais.

En I967, la totalité du produit est une première fois séchée à I $35^{\circ} \mathrm{C}$ pendant 30 à 35 minutes car les composants morphologiques de la panouille ont des taux de matière sèche très différents les uns des autres; de plus le hachage à l'ensileuse entraîne une grande hétérogénéité dans le volume des particules, les plus grosses (rafles) étant de surcroît les plus humides. Après ce premier séchage, les grains isolés qui constituent la grande majorité, sont alors séparés et le reste passe une seconde fois dans la déshydrateuse ( $10^{\circ} \mathrm{C}, 30^{\circ}$ minutes).

En I968, l'ensileuse utilisée assure un hachage plus efficace et fournit un produit beaucoup plus homogène par la taille de ses particules. Le séchage dure une heure à $\mathrm{I} 30^{\circ} \mathrm{C}$.

\section{4. - Analyses biochimiques et bactériologiques}

Les teneurs en matière sèche sont déterminées par séchage durant 24 heures dans une étuve ventilée réglée à $80^{\circ} \mathrm{C}$. Dans le cas des ensilages, la teneur en matière sèche est corrigée (MSC) de la perte des acides gras volatils, de l'ammoniaque et d'alcool par la relation établie par FATIANoFF et Govet (1969). Sur les échantillons secs broyés on détermine les matières minérales et la cellulose Weende.

Les microflores anaérobies sporulées sont dénombrées à partir d'échantillons frais non broyés selon la technique et avec les milieux au lactate ou à la gélatine de RosenBERGER (I95I). 
Le pH est mesuré sur le jus d'une macération au dixième après $\mathrm{I}_{5}$ heures à $4^{\circ} \mathrm{C}$. Les acides gras volatils (acétique, butyrique, propionique) sont dosés par chromatographie en phase gazeuse (Zelter, Leroy, I958), l'acide lactique par la méthode de Barnerr (I95I) et l'alcool par la micro-méthode de Nicloux (I93I). On dose aussi l'azote soluble total, l'azote ammoniacal (Conway, I950) et l'azote uréique (Conway, I950).

Toutes ces analyses sont effectuées sur des échantillons frais congelés dans l'azote liquide pour permettre un broyage suffisamment fin au Turmix. Cette opération est rendue nécessaire par l'hétérogénéité du produit (paille, grain, spathes, rafles) et la faible prise utilisée pour les analyses.

\section{RÉSULTATS}

\section{I. - Bilan agronomique}

Quantité de matière sèche récoltée à l'hectare.

Orge.

En I 967 , on ne constate pas de verse, la hauteur des tiges sur pied est homogène (95-100 cm) mais la longueur des pailles récoltées varie de I à $42 \mathrm{~cm}$ avec une moyenne de $19,3 \mathrm{~cm}$. Le rendement en grain immature (à o p. Ioo d'humidité) $(38,5 \mathrm{q} / \mathrm{ha}$ ) représente $9 \mathrm{I}, 2 \mathrm{p}$. IoO du grain récolté dix jours après à la moisonneuse-batteuse $(42,3 \mathrm{q} / \mathrm{ha})$ et la matière sèche totale au stade immature II $6,5 \mathrm{p}$. IOO $(49,2 \mathrm{q} / \mathrm{ha})$ (fig. 2).

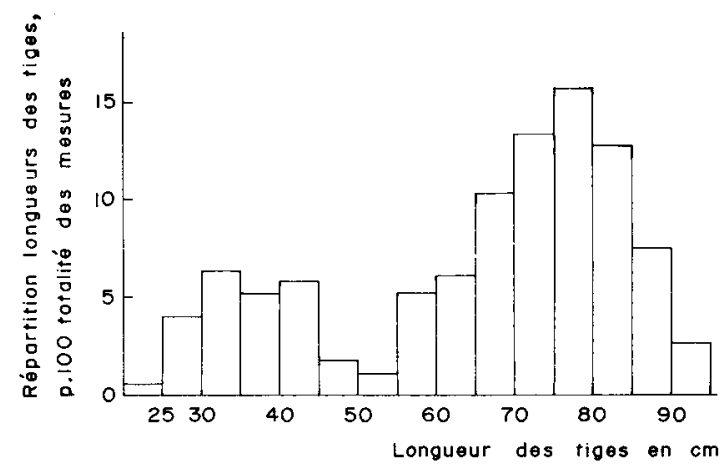

Figr. I. - Répartition de la longueur des tiges de l'orge récoltée en 1968 (en p. Ioo du nombre total de mesures)

En I 968 , le développement irrégulier des talles entraîne une grande variation dans la hauteur des tiges qui est en moyenne de $66 \mathrm{~cm} ; 23 \mathrm{p}$. Ioo de celles-ci mesurent moins de $45 \mathrm{~cm}$ et échappent en partie à la coupe (fig. I). Cette raison, et aussi une verse partielle mais néanmoins importante $(35 \mathrm{p}$. IOO) rendent difficile la coupe haute et régulière, et obligent à pratiquer une coupe moyenne. La longueur moyenne des pailles est pour cette récolte de $3 \mathrm{I} \mathrm{cm}$, les extrêmes allant de 5 à $60 \mathrm{~cm}$. Comparativement au grain mûr récolté huit jours plus tard (43,6 q MS/ha) les rendements ne sont que de $70 \mathrm{p}$. Ioo pour le grain immature (30,6 q MS ha) et $97 \mathrm{p}$. Ioo pour l'ensemble paille et grain (42,2 q MS/ha) (fig. 2).

Les taux de matière sèche du grain immature sont de $56,8 \mathrm{p}$. Ioo (Ig67) et 66, o p. 100 (Ig68) (fig. 3). 

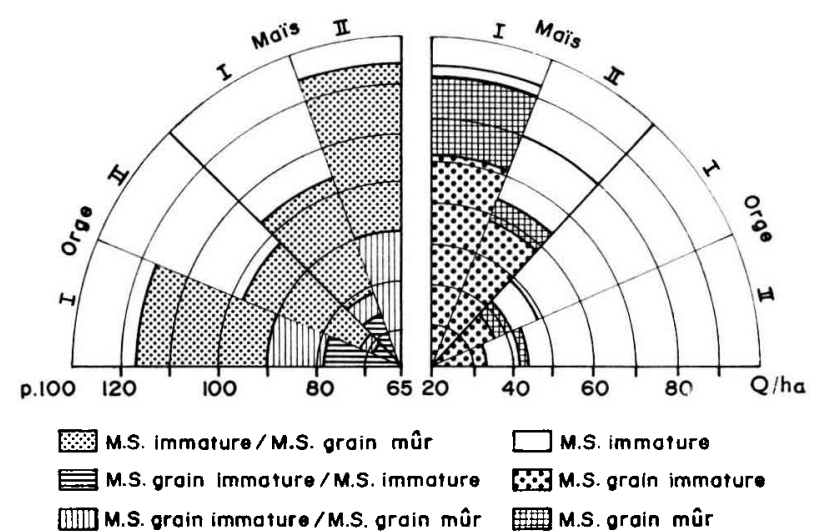

$\begin{array}{ll}\text { I Récolte } & 1967 \\ \text { II Récolte } & 1968\end{array}$

FIG. 2. - Rendements de matière sèche totale immature, grain immature et grain mûr Rapports relatifs de ces rendements

Maïs.

Les rendements totaux (fig. 2) en matière sèche au stade immature $(95,0$ et $79,8 \mathrm{q} / \mathrm{ha}$ ) excèdent ceux du grain mûr (9I,5 et $64,0 \mathrm{q} \mathrm{MS} / \mathrm{ha})$ de $4 \mathrm{p}$. Ioo en I 967 et $25 \mathrm{p}$. Ioo en I968. Cette différence est essentiellement due à ce que la récolte s'est faite en I968 entièrement à la main et a permis de conserver la totalité des spathes. Quant au rendement en matière sèche du grain immature, il ne représente que 80 p. Ioo à 90 p. Ioo du rendement en grain mûr. Les taux de matière sèche du grain immature sont de 62 , I p. Ioo (I967) et 6 I,3 p. Ioo (I968) et ceux du grain mûr respectivement de 68 p. Ioo et 69 p. I0o.

Proportion des différents constituants morphologiques.

Orge.

La proportion de matière sèche de paille récoltée passe de II,9 p. Ioo de la totalité en I967 à I8,2 p. Ioo en I968, celle du grain baisse de 78,3 p. Ioo à 72,7 p. Ioo et celle des barbillons et rachis varie peu (fig. 3). Il s'ensuit que la proportion de cellulose provenant du grain diffère d'une récolte à l'autre (fig. 4) ; en I967, elle représente 39,5 p. Ioo alors que la paille en apporte 38 , I p. Ioo. En I968 on a respectivement 26 p. Ioo et 53,9 p. Ioo. De même 85,3 p. Ioo (I967) et 86,9 p. Ioo (Ig68) de l'azote se trouvent dans le grain seul.

Mais.

En I 967 , selon que la récolte est faite à la main ou à la machine, la proportion de spathes passe de 8,7 p. Ioo à 2,5 p. Ioo et celle de barbes et pédoncules de 2,7 p. Ioo à 0,9 p. IOo ; ceci accroît corrélativement la proportion de grain (70,9 p. Ioo et $8 \mathrm{r}, 4$ p. roo (fig. 3). A technique de récolte identique (main) et d'une année sur l'autre il apparaît très peu de différence dans la répartition des constituants morphologiques: pour des taux de MS identiques (62,I p. Ioo et $6 \mathrm{I}, 3 \mathrm{p}$. IOo) le grain représente 70,9 p. Ioo et $73,2 \mathrm{p}$. Ioo de la MS récoltée et la rafle I6 p. Ioo et I 8 p. Ioo (fig. 3). 


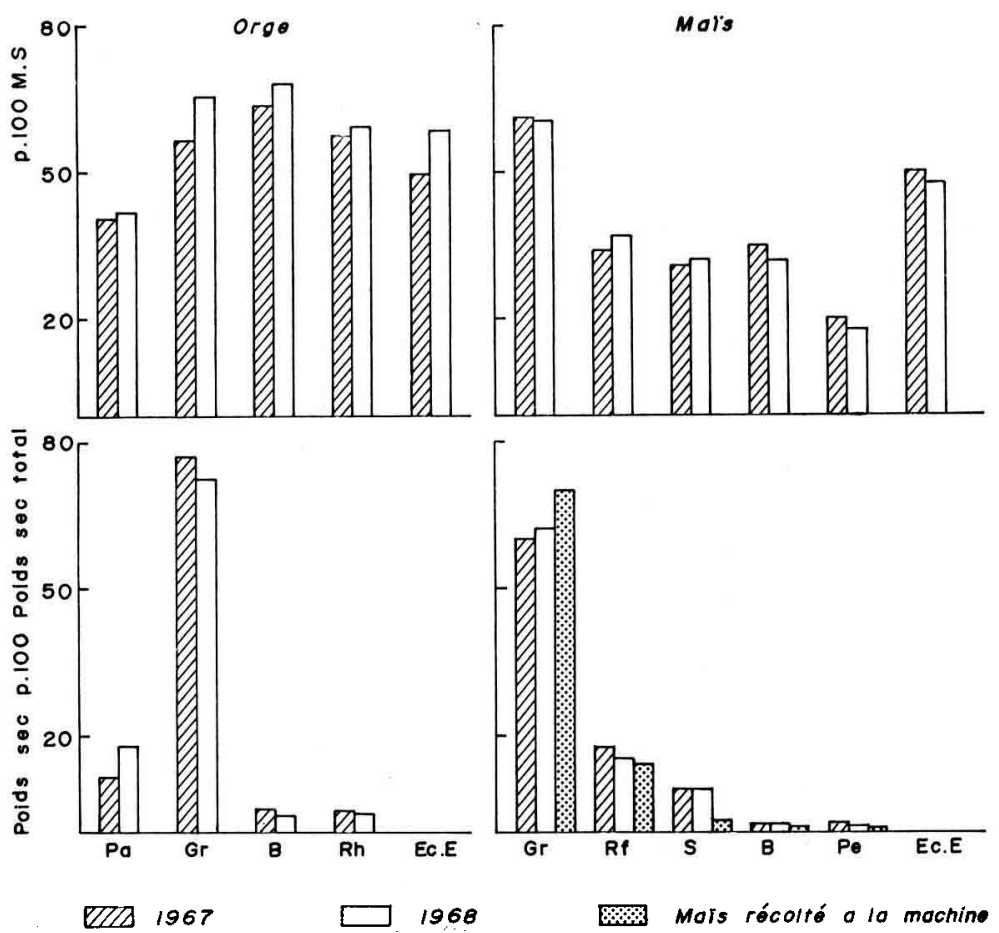

FIG. 3. - Pourcentage de matière sèche des différents composants morphologiques et répartition de ces constituants (poids sec p. roo du poids sec total)

$\mathrm{Pa}=$ Paille $; \mathrm{Gr}=$ Grain $; \mathrm{B}=$ Barbillons ou Soies $; \mathrm{Rh}=$ Rachis $; \mathrm{Rf}=$ Rafles $; \mathrm{S}=\mathrm{Spathes} ; \mathrm{Pe}=\mathrm{Pé}-$ doncules; $\mathrm{Ec} \mathrm{E}=$ Échantillon entier.

TABLEAU 3

Composition chimique des constituants morphologiques des céréales récoltées (p. Ioo MS)

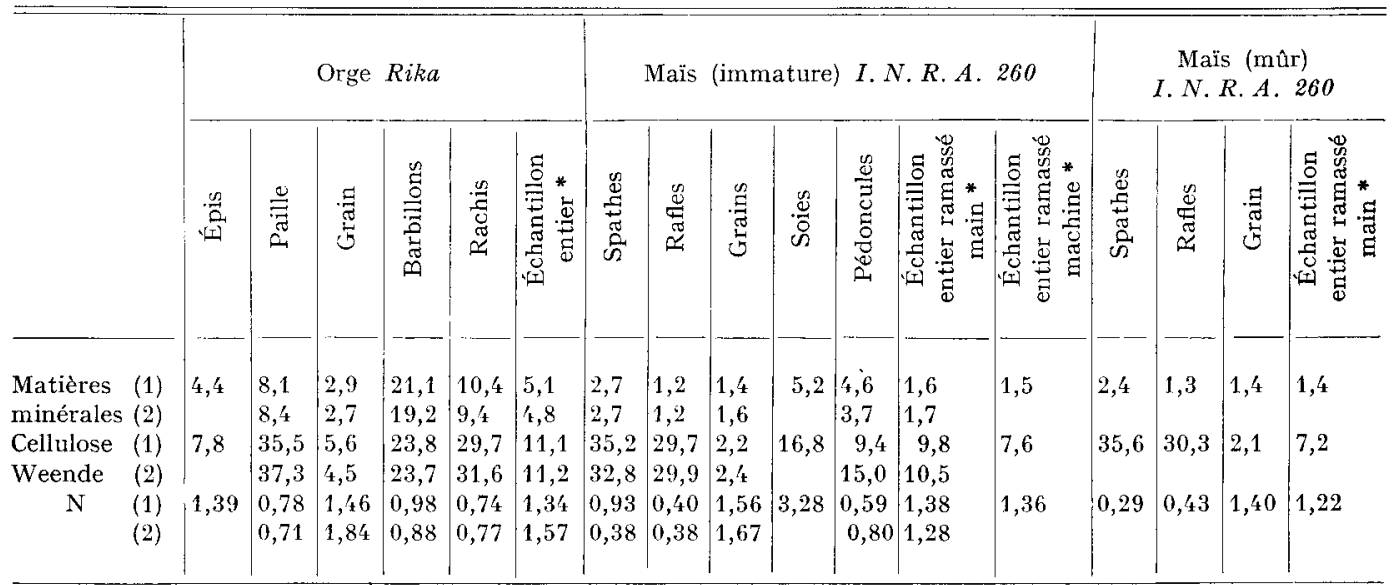

* Pour ces échantillons, les résultats sont calculés à partir des analyses de chaque constituant.

(1) 1967.

(2) 1968 . 
Les teneurs en cellulose brute et matières minérales de l'échantillon entier prélevé sur la fraction récoltée à la machine sont plus faibles que celles qui proviennent de la récolte manuelle (tab1. 3). Il ressort aussi de ce tableau que les différences dans les taux de cellulose et d'azote des constituants morphologiques de la panouille de maîs immature et mûr sont insignifiantes.

L'azote provient pour 85, I p. Ioo et 9I p. roo du grain (fig. 4) mais l'origine de la cellulose est plus diversifiée : 5I,9 p. Ioo et 49, I p. Ioo par la rafle, 29,7 p. Ioo et 29,3 p. Ioo par les spathes, I5, I p. Ioo et I 8 , I p. Ioo par le grain.
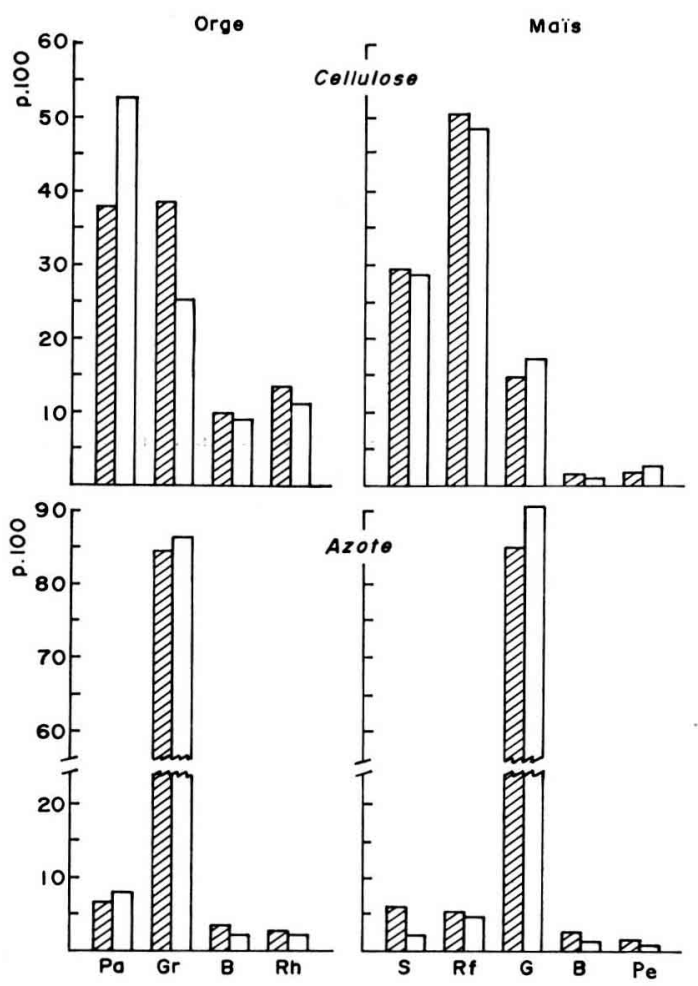

ED 1967

FIG. 4. - Proportions de cellulose et d'azote introduits dans l'aliment par chaque constituant morphologique de la plante

$\mathrm{Pa}=$ Paille $; \mathrm{Gr}=$ Grain $; \mathrm{B}=$ Barbillons ou Soies $; \mathrm{Rh}=$ Rachis $; \mathrm{S}=$ Spathes $; \mathrm{Rf}=\mathrm{Rafle} ; \mathrm{Pe}=\mathrm{Pe} \cdot$ doncules.

\section{2. - Conservation par ensilage}

Température.

En 1967 la température relevée au centre des silos d'orge en butyl lors de la mise en silo est de $37^{\circ} \mathrm{C}$. Après un mois elle s'abaisse à $26^{\circ} \mathrm{C}$ et après trois mois à $\mathrm{I} 9^{\circ} \mathrm{C}$. Dans le silo couloir d'orge cette diminution se fait plus lentement puisque pour les mêmes durées, elle est encore de $30^{\circ} \mathrm{C}$ et $25^{\circ} \mathrm{C}$.

En I968, la température des ensilages d'orge qui est à l'origine de $25^{\circ} \mathrm{C}$ augmente 
de 3 à $4^{\circ} \mathrm{C}$ durant les vingt premières heures. Elle se stabilise pendant 2 à 3 jours puis décroît ensuite régulièrement tout en suivant les variations de la température ambiante. L,e fond des silos, mieux tassé, se trouve souvent à une température plus faible $\left(3\right.$ à $6^{\circ} \mathrm{C}$ ) et $1^{\prime}$ on note aussi un refroidissement plus rapide dans l'orge $U^{-}$que dans $U^{+}$: la couche inférieure est à $23^{\circ} \mathrm{C}, \mathrm{I}^{\circ}{ }^{\circ} \mathrm{C}, \mathrm{II}^{\circ} \mathrm{C}$, après 30,60 et 95 jours de conservation dans $\mathrm{U}^{-}$alors que la même couche se trouve à $22^{\circ} \mathrm{C}, 20^{\circ} \mathrm{C}, 16^{\circ} \mathrm{C}$ dans $\mathrm{U}^{+}$.

Dans les ensilages de maïs la température initiale $\left(22^{\circ} \mathrm{C}\right)$ ne s'élève pas et se situe toujours au-dessous de celle observée dans les ensilages d'orge. Il faut évidemment tenir compte, dans cette comparaison, de la période de récolte du maïs (septembre) et de la proximité de l'hiver. En outre, la température n'est pas influencée par l'apport d'urée.

Concentration en métabolites fermentaires.

Orge.

Les concentrations en acides, alcool, $\mathrm{N}-\mathrm{NH}_{3}$, sont toujours très faibles et caractéristiques des ensilages à haute teneur en matière sèche (tab1. 4). D'une année sur l'autre, les résultats obtenus ne sont pas rigoureusement identiques, car en I968 le

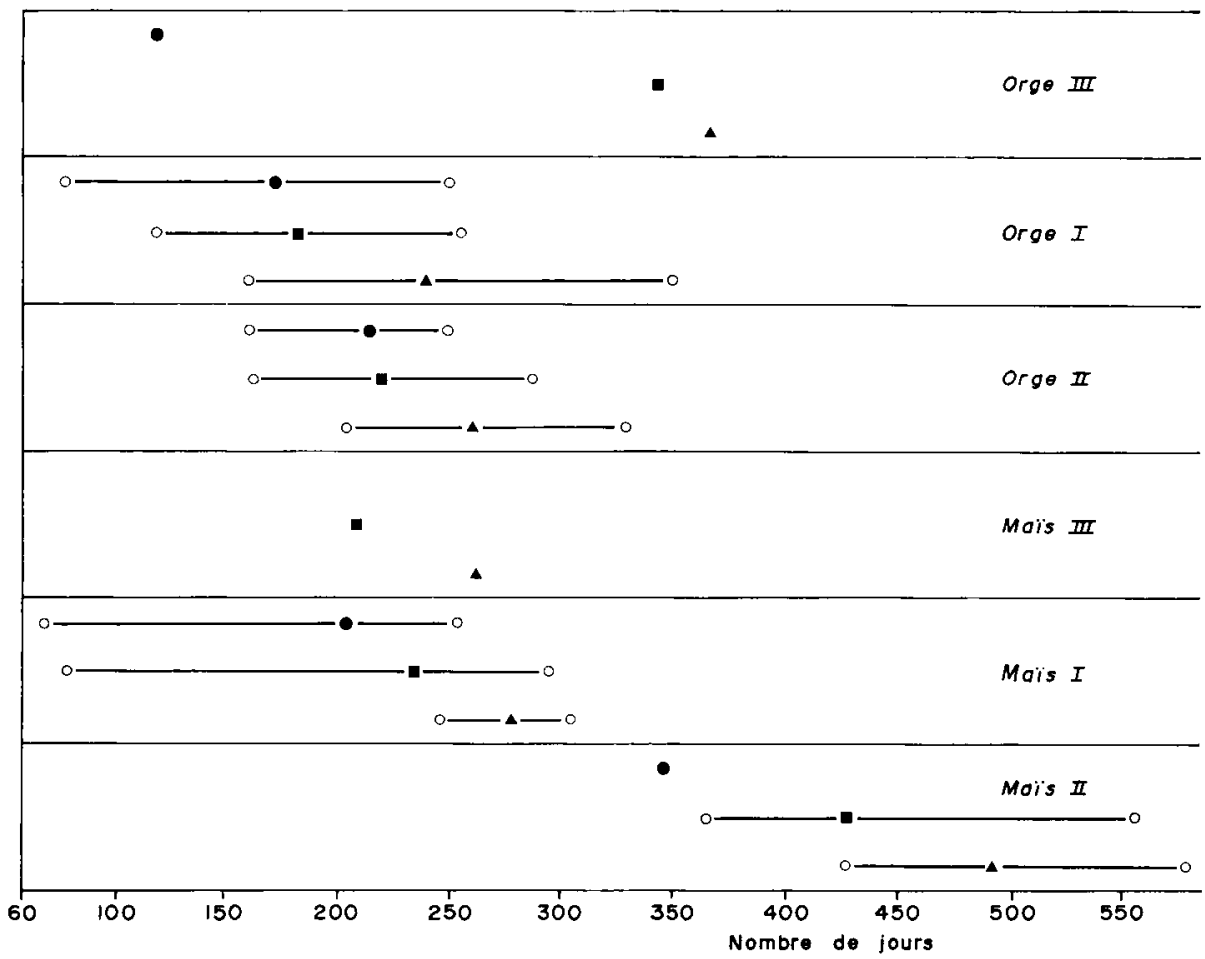

FIG. 5. - Durée de conservation et d'utilisation des ensilages

$I=$ Silo couloir $;$ II = Récolte I967; III = Récolte 1968

- Durées extrêmes a * Durées moyennes

$\circ-\cdots$ - Intervalle mise en silo - sondage

- _ - o Intervalle mise en silo — début désilage

$\mathrm{O}-\ldots$ - 0 Intervalle mise en silo - fin de désilage 
hachage est très fin, la température plus faible, la proportion de paille plus élevée et le taux de matière sèche du grain atteint $66 \mathrm{p}$. Ioo contre 56,8 p. Ioo en I 967 . De plus, les durées de conservation jusqu'à la prise des échantillons sont inégales (fig. 5).

Pour cette seconde série d'ensilages on note seulement une légère diminution des concentrations en acides lactique, propionique, butyrique, alcool, $\mathrm{N}-\mathrm{NH}_{3}$ et corrélativement une augmentation du $\mathrm{pH}$. Seul le taux d'acide acétique s'est faiblement accru. Les résultats des deux expériences sont toutefois suffisamment homogènes pour être groupés et mettre en évidence l'effet de l'urée (tab1. 4). L'addition d'urée a souvent un effet hautement significatif sur les fermentations : le $\mathrm{pH}$ y est en moyenne supérieur d'une unité à celui de $U^{-}(5,98$ contre $4,88 p<0,0 I)$ les concentrations en acides lactique ( $\mathrm{I}, 24 \mathrm{p}$. Ioo contre $\mathrm{I}, 85 \mathrm{p}$. Ioo $p<0,0 \mathrm{I})$, acétique $(0,42$ p. I0o contre 0,92 p. I0o $p<0,01)$, propionique $(0,04$ p. Ioo contre 0,08 p. IOO $p<0,0 \mathrm{I})$, butyrique $(0,4 \mathrm{I} \mathrm{p}$. Ioo contre $0,6 \mathrm{I}$ p. Ioo non significatif) sont plus élevés dans $\mathrm{U}^{+}$mais celles d'alcool sont comparables : I, II p. Ioo de la matière sèche corrigée dans $\mathrm{U}^{+}$et $\mathrm{I}, 27 \mathrm{p}$. Ioo dans $\mathrm{U}^{-}$. Dans les ensilages $\mathrm{U}^{+}$, l'azote uréique qui représente, à la mise en silo, 3I,4 p. Ioo de l'azote total s'abaisse à 4,8 p. Ioo le jour du sondage. Inversement il semble bien que la majorité de l'urée soit dégradée en ammoniac car le taux d'azote ammoniacal qui est de I p. Ioo dans la céréale au moment de la récolte atteint $24,6 \mathrm{p}$. Ioo en fin cle conservation soit cinq fois plus que dans $U^{-}(24,6$ contre $4,5 p<0,01)$.

\section{Mais.}

Alors qu'en I967, la durée de conservation au jour du sondage s'échelonne entre 67 et 25 I jours, elle est en I 968 de 346 (fig. 5). Or, si les résultats concordent globalement d'une année sur l'autre aussi bien pour les ensilages $\mathrm{U}^{+}$que $\mathrm{U}^{-}$, on observe néanmoins des différences importantes en particulier dans les concentrations d'acides, qui obligent à traiter statistiquement les deux expériences séparément (tabl. 4). Dans l'ensemble l'addition d'urée a les mêmes conséquences que pour l'orge mais les différences enregistrées par rapport au témoin $\mathrm{U}^{-}$sont ici peu importantes. C'est ainsi qu'en I967 un seul des huit résultats est significatif (azote soluble $p$ o,oI) ; en Ig68 les différences sont significatives pour le $\mathrm{pH}$, les concentrations en acide lactique et alcool qui augmentent dans $\mathbf{U}^{+}$. Par contre, l'apport d'urée n'a pas d'effet sur la concentration en acide acétique $(0,60 \mathrm{p}$. Ioo contre 0,63 p. Ioo en I 967 et $0,94 \mathrm{p}$. Ioo contre I,O0 p. Ioo en I968), et l'acide butyrique n'est présent qu'à l'état de traces malgré la durée de la conservation.

L'azote uréique qui représente $4^{8} \mathrm{p}$. Ioo de l'azote total à la mise en silo s'abaisse à 20-28 p. Ioo au moment du prélèvement de l'échantillon. L'accroissement du taux de $\mathrm{N}^{-} \mathrm{NH}_{3}$ dans $\mathrm{U}^{+}$en $\mathrm{I} 967$ et I968 n'est pas significatif et de plus, n'est pas proportionnel à la diminution de la concentration en urée.

Les ensilages d'orge et de mais obtenus en silo couloir lors de la première expérience et sans adjonction d'urée se distinguent des précédents par des taux de matière sèche plus faibles et surtout par des concentrations plus élevées en acides acétique et butyrique et en azote ammoniacal. 


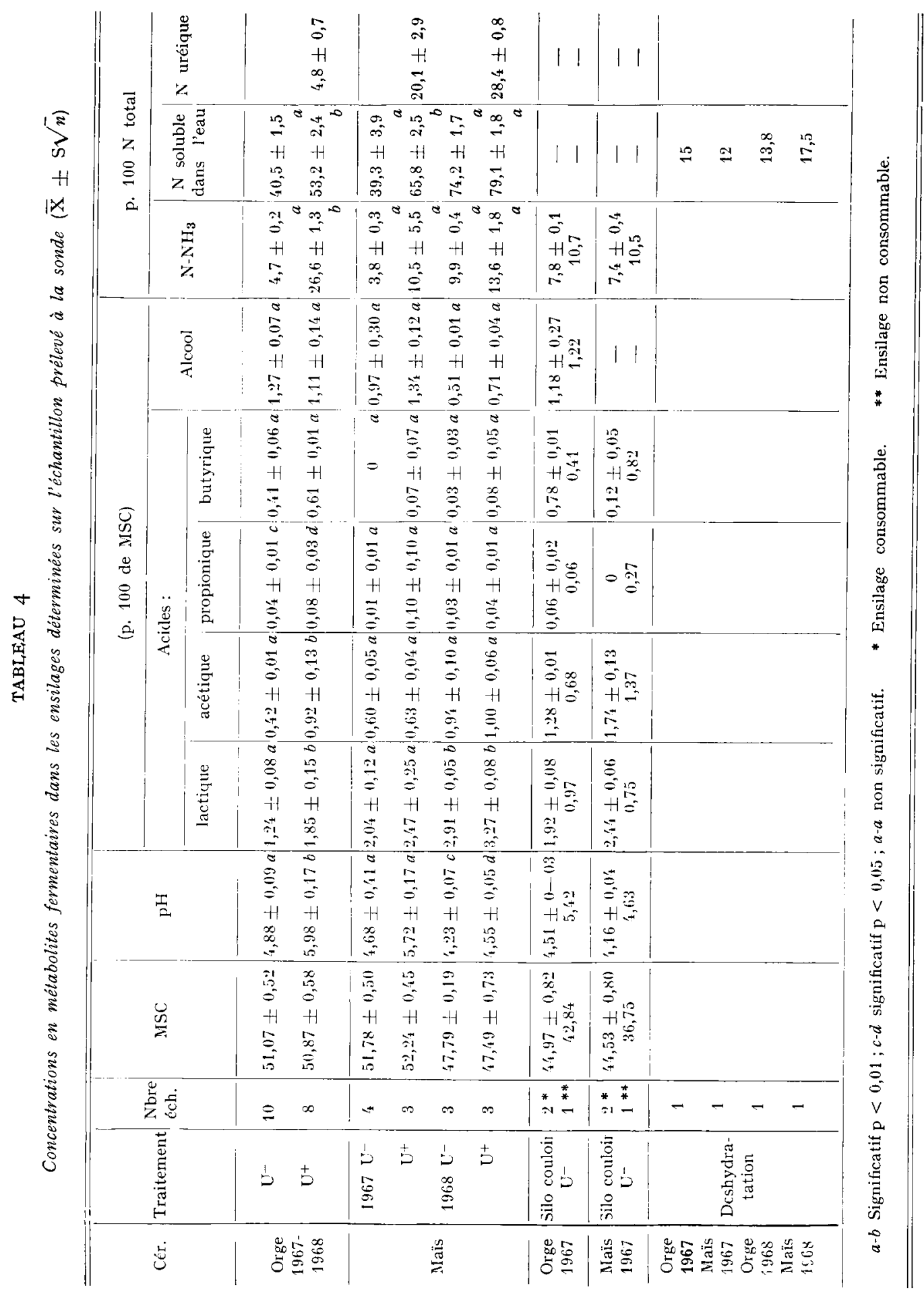


Dénombrement des microflores anaérobies sporulées.

Cette flore a été dénombrée à dessein car elle est responsable dans les ensilages de fourrage de la fermentation de l'acide lactique en acide byturique et de l'ammoniogenèse.

Dans la microflore anaérobie sporulée des ensilages d'orge $\mathrm{U}^{-}$les espèces fermentant le lactate (lactate + ) sont en nombre importants et dominent de ro à IOO fois les espèces protéolytiques ( $\mathrm{Pr}+$ ) (fig. 6). L'addition d'urée augmente la microflore $\mathrm{Pr}+$ alors que la microflore lactate + reste comparable. Dans les ensilages de maïs les nombres de spores lactate + et Pr + sont très faibles (Io à I ooo par gramme) et nettement inférieurs à ceux observés dans l'orge. L'urée n'entraine pas dans ce cas les augmentations enregistrées avec l'orge.
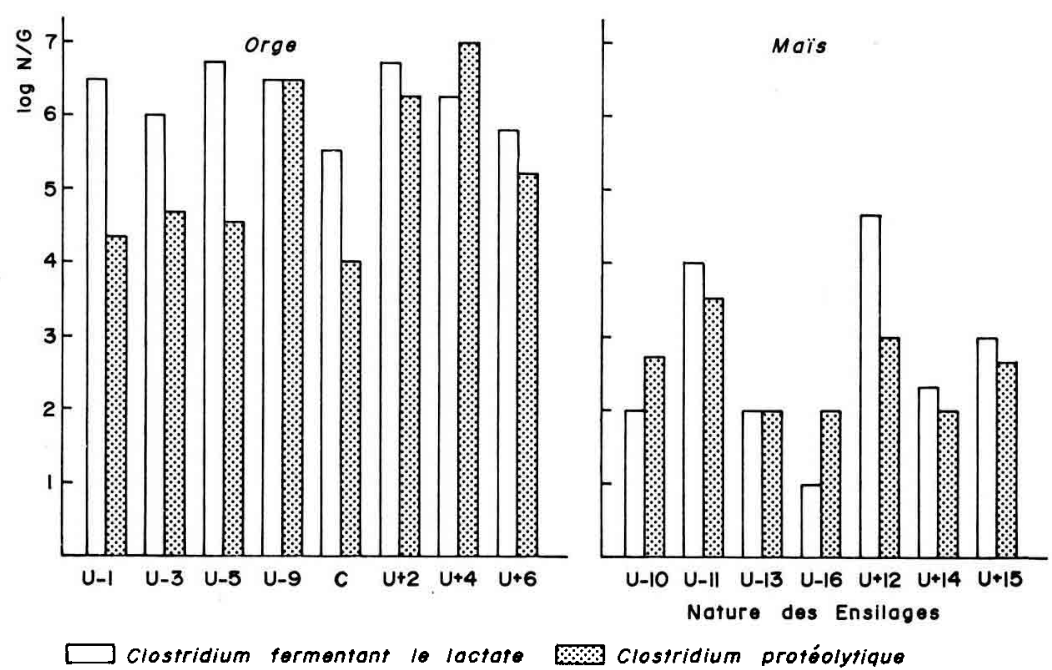

FIG. 6. - Nombre de spores de Clostridium dans les ensilages d'orge et de maïs (1967) $\mathrm{C}=$ Silo couloir $\quad \mathrm{U}+=$ avec urée $\quad \mathrm{U}-=$ sans urée

\section{Bilan de la conservation par ensilage.}

Dans les ensilages d'orge, conservés en silo de butyl les pertes augmentent de façon significative en présence d'urée. Pour la matière sèche on passe de $4,3\left(\mathrm{U}^{-}\right)$ à $9,4\left(\mathrm{U}^{+}\right)$et pour la matière organique de $5,0\left(\mathrm{U}^{-}\right)$à $\mathrm{IO}, 3\left(\mathrm{U}^{+}\right)($tabl. 5$)$.

Dans les mêmes silos, aucune différence n'apparaît pour les ensilages de maîs entre $\mathrm{U}^{+}$et $\mathrm{U}^{-}$et les pertes de matière sèche ou de matière organique se situent en moyenne entre $2 \mathrm{p}$. IOo et $4,5 \mathrm{p}$. IOO.

Bien que la qualité des ensilages obtenus en silo couloir soit très bonne, les pertes d'ensilage dues aux fermentations, quadruplent (I9 p. Ioo et 26 p. Ioo de la matière sèche ensilée) par rapport à celles des silos étanches. Il faut y ajouter une perte de I3,9 p. Ioo et I8,6 p. Ioo de la matière sèche consécutive à une exploitation beaucoup trop lente qui a favorisé l'échauffement et le développement de moisissures. 


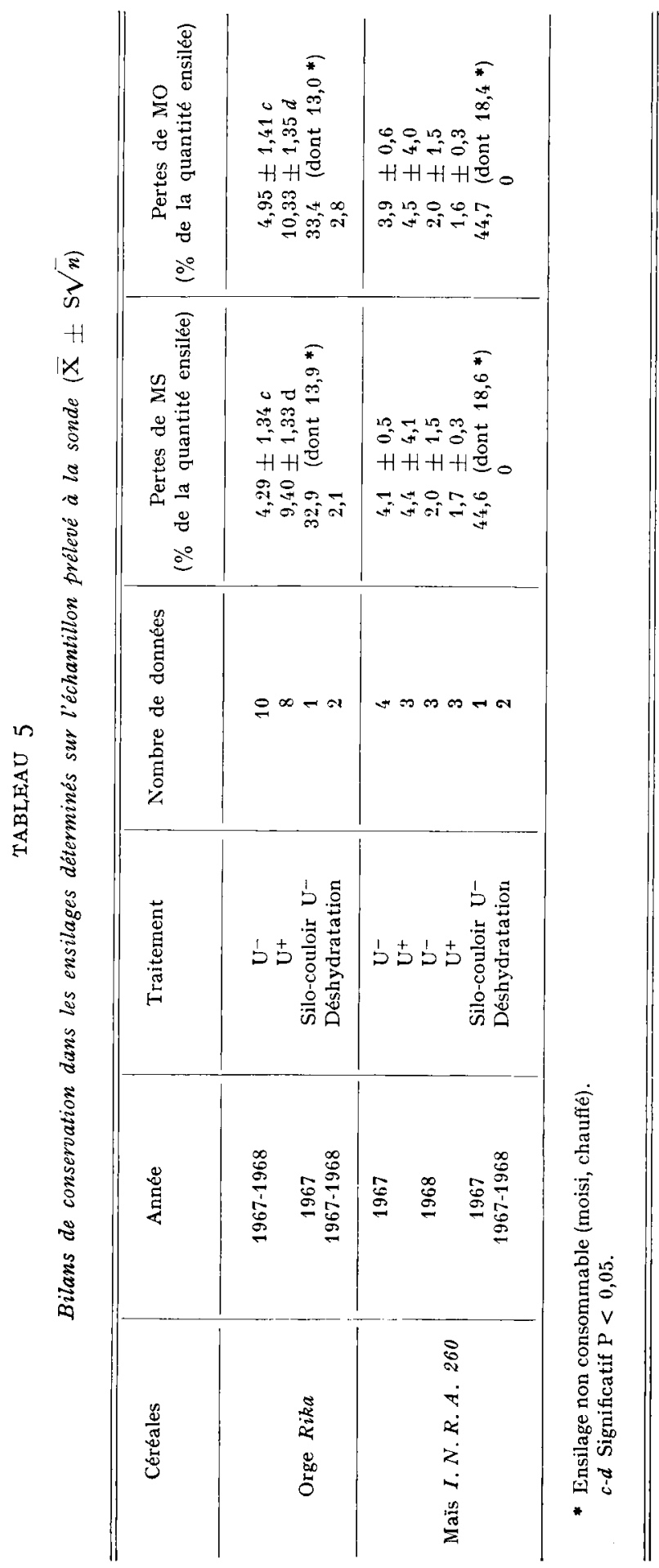




\section{3. - Déshydratation}

Compte tenu d'une teneur relativement homogène de la matière sèche entre les différents constituants morphologiques et de la structure creuse de la paille, l'orge se comporte beaucoup mieux à la déshydratation que le maiss. Pour ce dernier le produit conditionné à l'ensileuse se présente de façon très hétérogène, aussi bien par la taille des morceaux (grains set1ls, grains attachés à la rafle, spathes de diverses longueurs) que par les taux de matière sèche des constituants ( $20 \mathrm{p}$. Ioo pour les pédonct1les et 60 p. Ioo pour les grains). Ces écarts importants ont donc nécessité en I967 un second séchage de la fraction la plus humide. Néanmoins les pertes de matière sèche restent très faibles et l'on atteint tout au plus 2 , I p. Ioo.

\section{DISCUSSION E'T CONCLUSION}

Les observations faites sur le champ au cours des deux récoltes d'orge et les résultats des mesures effectuées montrent que la coupe haute est très délicate à réaliser dans la pratique. En effet, les tiges qui peuvent être de longueurs très différentes et la verse, fréquente chez 1'orge, rendent illusoire la régularité de cette coupe. Ces rendements pourraient être nettement améliorés en coupant entre ro et $85 \mathrm{~cm}$ du sol, mais aucune machine n'offre actuellement cette possibilité car l'amplitude maximum de variation de la hauteur de coupe pour une ensileuse hacheuse chargeuse tractée est d'une trentaine de centimètres. On peut toutefois modifier par des artifices la hauteur de la coupe mais non son amplitude; aussi pour couper au plus haut à $85 \mathrm{~cm}$ il ne sera plus possible de descendre au-dessous de $55 \mathrm{~cm}$ et de récolter l'orge versée. Ceci fut en partie le cas de la récolte I $968(35-65 \mathrm{~cm})$ et permet d'expliquer le faible rendement. Il semble donc bien que la combinaison d'une coupe moyenne $(40 \mathrm{~cm})$ avec une coupe basse $(\mathrm{IO} \mathrm{cm})$ dans les secteurs où la céréale a versé permette le gain le plus important d'UF par rapport à la récolte en grain (DEMARQUILLY, Paguet, Andrieu, ig69). Cette solution présente en outre l'avantage de fournir un aliment dont le taux de cellulose peut être adapté suivant le type d'animaux à nourrir.

Compte tenu des faibles dimensions des surfaces témoins et de l'hétérogénéité des parcelles, les rendements de maïs obtenus doivent être considérés avec réserve. Néanmoins, il faut noter qu'au cours des deux expériences les rendements en grain sont nettement plus faibles au stade immature.

Dans les ensilages, les concentrations en métabolites fermentaires sont faibles et caractéristiques des ensilages à teneur en matière sèche élevée. Si la qualité des ensilages est généralement bonne, le maïs se comporte toutefois mieux que l'orge qui semble plus sensible à la fermentation butyrique (EDwards, Donalson, de MC GREGOR, I968; MC GREGOR, EDWARDS, I968) et qui tirerait peut-être profit l'adjonction d'un conservateur tel que l'acide formique ou propionique.

I1 faut cependant tenir compte dans la comparaison orge-mais du fait que ce dernier, conditionné au broyeur à marteaux, se trouve réduit en très fines particules, alors que l'orge est hachée à l'ensileuse. Ainsi pour cette dernière, la paille est tron- 
çonnée mais non éclatée, et les grains ne sont pas fragmentés. Le tassement est aussi rendu plus difficile comme en témoignent les densités $\left(250 \mathrm{~kg}\right.$ de $\mathrm{MS} \mathrm{au} \mathrm{m}^{3}$ pour l'orge et $35^{\circ} \mathrm{kg}$ pour le mais) et l'élévation de température dans les silos d'orge.

L'enrichissement de la céréale en azote uréique active les fermentations sans faire apparaître toutefois des concentrations d'acide butyrique ou d'azote ammoniacal importantes. Dans le cas de l'orge $\mathrm{U}^{+}+$la quasi-totalité de l'azote uréique est retrouvée en fin de conservation sous forme ammoniacale alors que pour le maîs Ucette compensation qui n'apparaît pas, est difficilement explicable.

Sur le plan technologique la conséquence la plus importante de l'incorporation d'urée dans l'orge réside dans l'augmentation des pertes de matière organique qui doublent et dont il est logique de penser qu'elles proviennent essentiellement de l'amidon qui est le glucide le plus facilement fermentescible de ce type d'ensilage. Dans cette hypothèse les pertes en matière sèche du grain calculée à partir des proportions trouvées dans l'ensilage seraient de I5 p. Ioo. Cet accroissement des pertes en présence d'urée a d'ailleurs déjà été remarqué (OWENS, MEISKE, GOODRICH, I969) pour les ensilages de maïs et il est évident qu'il doit être relié à l'activité fermentaire plus intense. Dans ces conditions et compte tenu de la haute valeur énergétique de l'amidon, il serait intéressant d'étudier l'action de formes d'urée moins faciles à hydrolyser.

En conclusion, la conservation, même prolongée, en silo étanche de la panouille de maïs immature broyée en présence d'urée ne présente pas de difficulté alors que celle d'une orge avec sa paille devrait être réexaminée soit en lui ajoutant un conservateur, soit en la récoltant plus précocement. Inversement, la déshydratation à basse température, si elle est aisée pour l'orge, l'est moins pour le mais en raison des différences importantes dans les vitesses de dessiccation et les taux de matière sèche de ses constituants morphologiques. Cette technique permet d'obtenir un produit stable avec des pertes minimes mais son coût est élevé.

Reçu pour publication en février 1971

\section{SUMMARY}

CONSERVATION BY ENSIIAGE OR DEHYDRATION

OF A VARIETY OF BARLEY AND OF MATZE

During 2 consecutive years, the yields were compared of a variety of barley (Rika) and of maize (INRA 260), harvested at the "watery kernels " stage, one to four weeks before maturity, and at the "ripe for cutting "stage. The barley was harvested with 20 to $30 \mathrm{~cm}$ of straw and the maize cobs with their shuck spathes. These cereals were conserved either by ensilage with urea $\left(\mathrm{U}^{+}\right)$or without urea $\left(\mathrm{U}^{-}\right)$, in impervious silos made of butyl, or by dehydration at low temperatures $\left(13 \mathrm{O}^{\circ} \mathrm{C}\right)$. The products of bacterial fermentation were analyzed in the ensiled material, and the efficiency of the conservation method was measured in all cases.

The amount of dry matter (DM) in the case of both cereals was 8.8 to $30 \mathrm{p}$. Ioo lower, depending on the experiment, at the "watery kernels "than at the mature stage. The dry matter of the immature grain was between $72 . x \mathrm{p}$. I 00 and $79 \mathrm{p}$. Ioo of the total dry matter harvested. The yield of the total dry matter harvested varied from $-8 \mathrm{p}$. Ioo to $24.8 \mathrm{p}$. 1oo. It is concluded that important losses result from the fact that a regular cut of an adequate height is difficult to obtain because of lodging, that occurs frequently with barley, and because it is difficult to adapt existing machines to this purpose. 
The proportions of the various morphological components (straw, grain, barbs, rachis, cob, peduncle) are given with their chemical compositions (organic matter, cellulose Weende, nitrogen).

The concentration of the metabolites of fermentation (lactic acid, volatile fatty acids, alcohol, $\mathrm{NH}_{3}$, nitrogen) were low in the silages, and were characteristic of high dry matter silages.

Barley silage is, nevertheless, more subject to butyric fermentation than maize silage, and the number of spores of lactate-fermenting or proteolytic Clostridium often attained $10^{6}$ to $5^{\circ} \mathrm{IO}^{7}$ per gram. The addition of urea resulted in an increase in the amount of ammonia and a significant increase in the intensity of fermentation. The losses in organic matter in the barley silages were doubled as a consequence of this (IO p. Ioo).

In the case of ensiled corn cobs, these losses were the same in $\mathrm{U}^{+}$and $\mathrm{U}^{-}$silages (2 p. Ioo to 3 p. 100$)$.

Dehydration at low temperatures is easily accomplished for barley, but less so for maize, owing to the important differences in the amount of dry matter and the rate of drying of the various morphological constituents. This technique allows a stable product to be obtained with the minimum of loss, but at a high cost.

\section{RÉFÉRENCES BIBLIOGRAPHIQUES}

Barnett A. J. G., I95I. The colorimetric determination of lactic acid in silage. Biochem. J., 49, 527528.

Conway E. J., 1950. Microdiffusion analyses and volumetric error, $4^{\mathrm{e}}$ Edit. Grosby Lockwood and son, London.

Demarquilly C., Paquet J., AndrieU J., I969. Les céréales immatures. Bull. tech. Inf. Ingrs Servs, agric., 244, I-2O.

Edwards R. A., Donaldson Elisabeth, Lc Gregor A. W., ig68. Ensilage of whole-crop barley. I. Effects of variety and stage of growth. J. Sci. Fd. Agric., 19, 656-66o.

Fatianoff Nathalie, Gouet $\mathrm{Ph}$, 1969. Relation permettant de corriger rapidement et avec précision la matière sèche des ensilages séchés à l'étuve. Ann. Zootech., 18, 407-4 I8.

Mc Gregor A. W., Edwards R. A., 1968. Ensilage of whole-crop barley. II. Composition of barley and barley silage at different stages of growth. J. Sci. Fd Agric., 19, 66I-666.

Nicloux L., I931. Recherches sur l'alcool éthylique. I. Microdosage. Bull. Soc. chim. Biol., 13,857-876.

Owens Frederic N., Leiske J. C., Goodrich R. D., i 969 . Effects of calcium sources and urea on corn silage fermentation. J. Dairy Sci., 52, I817-1822.

Rosenberger R. F., i95I. The development of methods for the study of obligate anaerobes in silage. Proc. Soc. Appl. Bact., 14, I6I-164.

Zelter S. Z., Leroy F., 1958. Azote uréique et activité bactérienne in vitro au niveau du rumen. Ann. Zootech., 3, 173-183.

Zelter S. Z., Charlet-Léry Geneviève, Tisserand J.-L., i97I. Influence chez le taurillon en croissance du traitement de conservation (ensilage ou déshydratation) de la céréale immature (orge, maiss) sur sa valeur nutritive et sur l'efficacité métabolique de l'urée ajoutée. Ann. Zootech., 20, r35-I52. 